\title{
3D Reconstruction of the NADH:Ubiqinone Oxidoreductase (Complex I) from Yarrowia lipolytica Lacking Subunit NUMM.
}

\author{
Christine T Nolan ${ }^{1}$, Katarzyna Kmita ${ }^{2}$,Volker Zickermann ${ }^{2}$, Teresa Ruiz ${ }^{1}$ and Michael Radermacher ${ }^{1}$ \\ 1. Department of Molecular Physiology and Biophysics, University of Vermont, Burlington VT, USA \\ 2. Institute of Biochemistry II, Medical School, Goethe University, Frankfurt/M, Germany
}

Complex I is the first enzyme of the respiratory chain in both the bacterial and the mitochondrial inner membranes. The mitochondrial enzyme from the aerobic yeast Yarrowia lipolytica has a molecular weight close to $1 \mathrm{MDa}$ and contains at least 42 subunits. Complex I in all species exhibits an L-shape with two arms of similar length, one embedded in the membrane (membrane arm) and the other reaching into the mitochondrial matrix or the bacterial cytoplasm (matrix arm). NADH binds to the outer protrusion of the matrix arm and is oxidized. Concomitantly electrons are transferred to ubiquinone and four protons are translocated across the membrane. The four protons contribute to the membrane potential that is used by the F-ATPase to produce ATP.

The assembly of complex I is an intricate process, mediated by multiple assembly factors [1]. In the past we have analyzed the effect of assembly factor N7BML (NDUFAF2), which is involved in the late stages of complex I assembly. The lack of this assembly factor still results in the formation of a close to fully functional complex I, albeit with a lower yield. The medium resolution 3D structure also showed no significant differences when compared to the holo enzyme [2]. Assembly factor N7BML binds transitory to complex I and therefore is difficult to localize. We have created a sub-complex, lacking subunit NUMM (13 kDa) [3]. Deletion of the NUMM gene compromised a late stage of complex I assembly that includes the stable insertion of iron sulfur cluster N4. Activity of complex I in this deletion mutant was reduced to $44 \%$. Complexome profiling revealed that subunit N7BM was also missing from the stalled sub-complex, while assembly factor N7BML (27.6 kDa) was found attached to the enzyme. The goal of this study was to localize the assembly factor and thus obtain a better understanding of this step in complex I biogenesis.

The structure of the $\triangle \mathrm{NUMM}$ subcomplex was analyzed by $3 \mathrm{D}$ electron microscopy using random conical tilt single particle techniques. Purified sample was applied to continuous-carbon coated grids at $0.02 \mathrm{mg} / \mathrm{ml}$ and deep stain embedded (2\% PTA, $\mathrm{pH} 7.2$ ). Tilt pairs were recorded at $\sim 58^{\circ}$ and $0^{\circ}$ under low dose conditions $\left(10 \mathrm{e}^{-} / \AA^{2}\right)$ at a $100 \mathrm{kV}$ and $52 \mathrm{kX}$ nominal magnification. Micrographs were digitized with a $7 \mu \mathrm{m}$ pixel size on a Zeiss SCAI flatbed densitometer. Transparencies were converted to optical densities, calibrated to the unexposed film background, and the resulting images were interpolated down to a final pixel size of $4.02 \AA$ on the specimen scale. A total of 26,600 image pairs were extracted and the $0^{\circ}$ images were processed in a sequence of: 1 ) centration; 2) single reference alignment; 3) correspondence analysis followed by classification; and 4) multi-reference alignment against the class averages. Steps 3 and 4 were iterated and ended with a final correspondence analysis and classification step that resulted in 8 classes. Tilt images were corrected for the microscope contrast transfer function as described in [4]. 3D structures were calculated using Radon inversion methods and the structures were refined using 3D reference based projection alignment as described in [5]. The resolution after each refinement step was assessed using the Fourier Shell correlation.

The $\triangle$ NUMM reconstructions reflect the high variability of the subcomplex. The 3D structures reveal a 
destabilization of the matrix arm, manifest most pronouncedly in the $\mathrm{N}$-module with either a weakened density in the lobe containing the $51 \mathrm{kDa} / 24 \mathrm{kDa}$ subunits or a loss of other matrix arm subunits. The lack of NUMM seems to predominantly destabilize the NADH binding region which would be consistent with its location adjacent to these subunits. The resolution of the 3D reconstructions is mainly limited by sample heterogeneity as evidenced by the interpretation of the shape of the Fourier Shell correlation curves and the CTF correction results. Currently we are improving pattern recognition and classification techniques to overcome this limit, which should lead to higher resolution structures that will permit to clearly recognize the position of the assembly factor in the subcomplex.

\section{References:}

[1] Mckenzie, M. \& Ryan, M. T. IUBMB Life 62, (2010) p. 497.

[2] Nolan, C. T. et al. Microsc. Microanal. 21, (2015), p. 901.

[3] Kmita, K. et al. Proc. Natl. Acad. Sci. 112 (2015), p. 5685.

[4] Radermacher, M. et al. J. Struct. Biol. 135, (2001), p. 26.

[5] Radermacher, M. Scanning Microsc. 11, (1997) p. 171.

[6] Petterson, E.F., Goddard, T.D., et al., J. Comput. Chem. 25 (2004), p.1605-1612.

[7] The authors acknowledge funding from NIH, grant 2RO1 GM068650 to M.R, and from DFG grants ZI 552/4-1 and EXC 115 to V.Z.
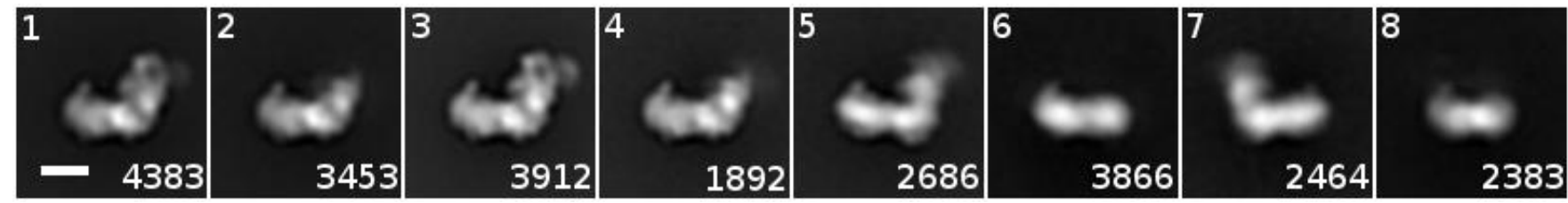

Figure 1. Class averages of the $0^{\circ}$ images after the last round of multireference alignment and correspondence analysis. Top indicator: class number, bottom indicator: class size. Scale bar $10 \mathrm{~nm}$.

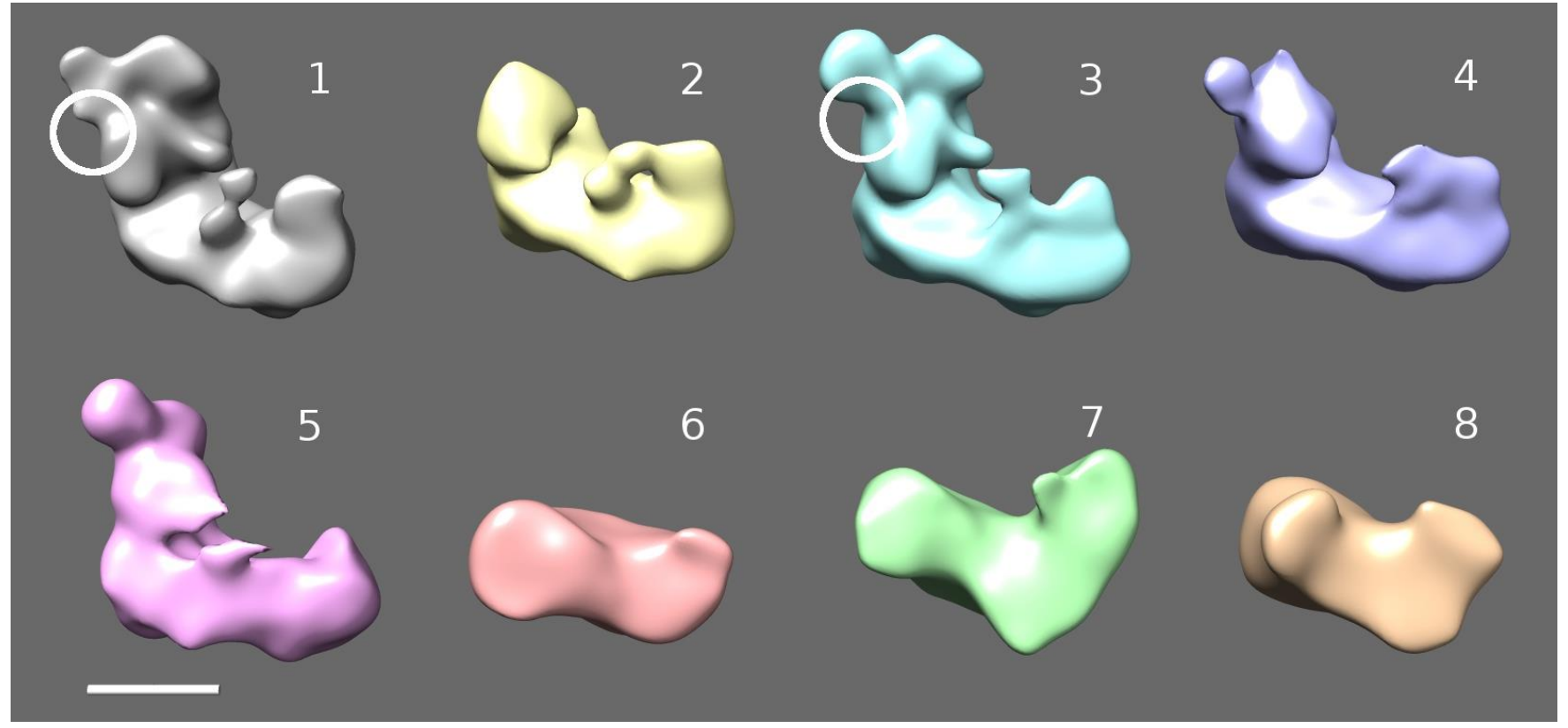

Figure 2. 3D reconstructions of the eight class averages. Circles indicate the putative location of N7BML and the missing NUMM subunit. Scale bar $10 \mathrm{~nm}$. 